\title{
Introducing PneuAct: Parametrically-Designed MRI-Compatible Pneumatic Stepper Actuator
}

\author{
Foad Sojoodi Farimani and Sarthak Misra
}

\begin{abstract}
Pneumatic stepper motors are one of the promising alternative actuation methods for motion control in environments where electromagnetic (EM) motors cannot be used. Due to the lack of commercial off-the-shelf products, researchers working on MR compatible robotics have to develop their own pneumatic actuators. This imposes extensive costs and delays on the development process. Additionally, the current solutions are limited in their range of specifications and are difficult to manufacture. In this paper, proof-of-concept-prototypes for a family of parametrically designed, electromagnetically stealth, rotational pneumatic stepper motors are presented. The main objective of the paper is to demonstrate a general purpose nonelectromagnetic actuation method, which can be customized and integrated into any design. Customizability, miniaturization, safety and affordability are some of the key features of the presented work. The developed prototypes are entirely 3D-printed and contain no sealing, bearing or lubrication. Thanks to the low production cost, the motor can be used as a disposable part in surgical applications. Experiments demonstrate effectiveness of the design in terms of cost-efficiency, versatility, MRIcompatibility, speed and performance. In order to optimize the design and control algorithm, empirical equations are presented describing response time of a pneumatic system to sequential pressure signals. A rotational speed of $800 \mathrm{rpm}$, total volume of $4.6 \mathrm{~cm}^{3}$ and resolution of $3^{\circ}$ are some of the design attributes. The effects of clearance on stick-slip effect and leakage in a 3D printed cylinder-piston are also presented.
\end{abstract}

\section{INTRODUCTION}

Electromagnetic (EM) stepper motors are "de facto standard" actuation method for motion control in automation and robotics. However, there are cases where EM motors are not applicable. Medical imaging modalities, like magnetic resonance (MR) and computed tomography (CT), are examples of such environments. Alternative actuation methods have been explored by different industrial and scientific groups [1]. Pneumatic power transmission systems are considered as a promising replacement [2]. In addition to having a high power-to-size ratio, pneumatic actuators are inherently non-EM. Particularly, for medical applications, compressed air is easily accessible in the hospital setting. Air does not interfere with imaging processes and clean air is in compliance with hygienic requirements. Therefore, pneumatic actuation could be the desirable method in image guided robotic surgery. Despite their advantages, pneumatic systems present complicated dynamic behaviors, including compressibility, viscosity, turbulence, throttling, friction and leakage. As a result, position control of servo-pneumatic

The authors are affiliated with Surgical Robotics Laboratory, Department of Biomechanical Engineering, University of Twente. S. Misra is also affiliated with the Department of Biomedical Engineering, University of Groningen and University Medical Center Groningen, The Netherlands.

\{f.s.farimani, s.misra\}@utwente.nl

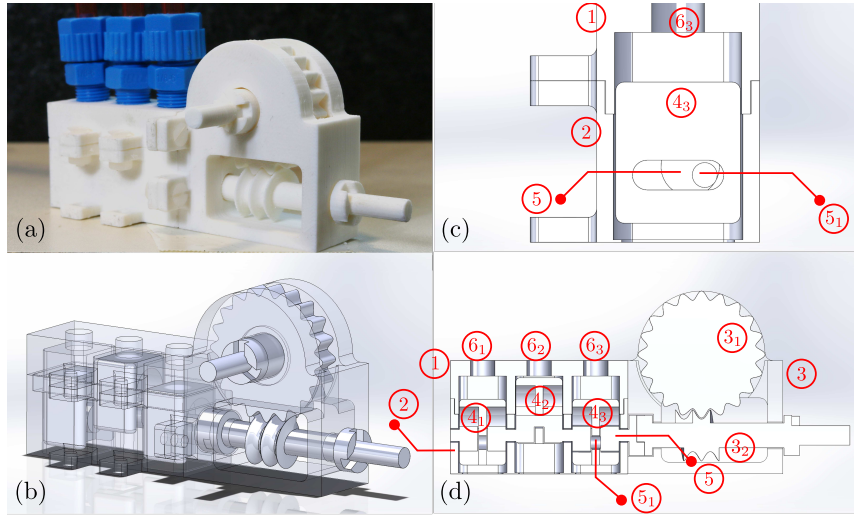

Fig. 1. (a) A 3D printed prototype of the PneuAct actuator. (b) Components: (1) cylinder head, (2) cylinder, (3) reduction gear-head, (31) pinion gear, (32) worm gear, (4) pistons, (5) crankshaft, (6i $(i=1,2,3)$ inlets. (c) A transverse cross section of the motor passing through piston (43 and crankpin (51). The crank-pin slides in the horizontal slot in the piston stem, converting the reciprocating motion to rotation (scotch yoke mechanism) (d) A longitudinal cross section of the motor illustrating the intermeshing mechanism between the crank shaft, the pistons and the worm drive.

actuators, such as cylinder-pistons, turbines and vane motors is challenging. In contrast, stepper motors are able to deliver high precision open-loop motion control with a reliable accuracy. A pneumatic stepper motor (PSM) is a volumetric fluidic actuator, able to sustain a speed synchronous with the exciting frequency, independent from the external torque and input pressure, within a certain range. This working range of PSMs is called a stall curve.

First rotational PSMs were reported by Suzumori et al. [3], [4]. The presented motors were not MRI compatible. They also included many parts from different materials resulting in high manufacturing costs. Taillant et al. were the first group to report a non-EM rotational PSM in a mechanism for image guided surgery [5]. The concept, called clockmaking principle, resembled a ratcheting socket wrench. The reported actuator was merged into the mechanism and cannot be considered as a general purpose pneumatic motor.

Stoianovici et al. introduced PneuStep, the electromagnetically stealth pneumatic stepper motor [6]. PneuStep demonstrated significant performance in terms of resolution and speed-torque characteristics. However, it comprised twenty five parts, made from several materials, imposing high fabrication costs. Their motor was also not back-drivable which is important for a safe surgical robotic system. A surgical motorized mechanism with self-locking actuators/joints, cannot be easily retracted, if an emergency stop should happen. 




Fig. 2. 3D printed proof-of-concept prototypes, generated form a single parametric CAD file with different dimensions. @ Motor with dimension $30 \times 20 \times 46 \mathrm{~mm}^{3}$. (b) Gearhead compatible with @ with a ratio of $1: 20$. (C) Motor with dimensions $30 \times 20 \times 55 \mathrm{~mm}^{3}$ (d) Gear-head compatible with (C) with a ratio of $1: 40$ (e) Motor with dimensions $40 \times 25 \times 40 \mathrm{~mm}^{3}$ (f) Gearhead compatible with (e) with a ratio of $1: 40$. (g) Motor with dimensions of $10 \times 15 \times 28 \mathrm{~mm}^{3}$, with a total volume of $4.6 \mathrm{~cm}^{3}$. It is the smallest bidirectional rotational pneumatic stepper motor among state of the art.

A safe surgical mechanism should have a passive mode. In the case of emergency, the mechanism will be deactivated and turned into a passive tool holder. Therefore, surgeons have full control over the operation at hand. Sajima et al. reported a novel concept of a PSM similar to a barrel engine [7]. In comparison to former designs, their concept had a smaller frontal area, as well as being more affordable. A major issue with this design is that the parts in the motor are not mechanically coupled. As a result, inaccuracy due to slipping is possible. Chen et al. reported a motor based on the conventional piston and crankshaft concept [8]. This was the first time a conventional engine concept was introduced in the field of MRI compatible PSMs. The motor had the advantage of using commercial off-the-shelve products. However, it was rather big, not monolithic and made from many different modules. Chen et al. later reported another motor similar to the mechanism used in push pens [9]. It was the smallest stepper motor at that moment. However, it was not bidirectional and in terms of mechanical performance, it was less effective than other presented motors.

This paper contributes to the field of pneumatic stepping actuation by providing a novel concept of a paramertically designed, assembly-printed pneumatic stepper motor Fig. 2. The motor is electromagnetically stealth and contains no sealing, bearing or lubrication. It is safer than former designs in terms of being back-drivable with adjustable frictional impedance. Travel time of a square pressure signal in a pneumatic tube is studied and empirical equations are presented. Effects of clearance on the amount of force generated in a 3D-printed cylinder-piston is studied. A rigid body model of the system is implemented to compare the calculated and measured torques in a motor. Results of the experiments are used to optimize the design and control system.

\section{DESIGN AND PROTOTYPE}

Mechanical composition of any PSM can be broken down to three different elements. The first part converts air pres- sure into mechanical work. The second part is a linear to rotational motion converter. The last component in a PSM is a reduction drive. The reduction drive depends on the user needs for back-drivability, resolution, size, torque and speed.

To address the shortcomings in the state-of-the-art, a novel design is presented. The presented concept includes a piston to harness air energy, combined with a scotch yoke mechanism to generate rotational motion. In comparison to normal crank-shaft, scotch yoke mechanism is easier to make, has a more robust structure and has less number of moving parts. The motor is parametrically designed from the ground up. As a result, the CAD model can be easily updated automatically by a limited number of driving parameters, such as required torque, pressure, speed, resolution, durability, working range and size. Motors presented in this paper are back-drivable with adjustable friction through parametrically controlled clearances. The mechanical design exploits the potential of additive manufacturing for non-assembly production, reducing costs to a high extent.

A prototype of the proposed design is illustrated in Fig. 1. The motor comprises three different modules printed separately. The main body includes the cylinder (2), the crankshaft (5) and three pistons (41), (42) and (43). The cylinder-head (1) is printed separately to ensure minimum possible clearance between the cylinder and the pistons. The gear-head module (3) includes three parts: structure, worm-gear (32) and pinion (31) all printed together. Pistons, are arranged linearly. As a result, the motor has a small and customizable frontal area. A schematic of the electro-pneumatic setup is shown in Fig. 3. Three solenoid pneumatic valves create alternating pressure signals to excite each piston. Consequently, the pistons drive the crankshaft through the scotch yoke mechanism.

To demonstrate an example of a reduction gearing, a worm-drive with sinusoidal tooth profile was prototyped. The gearhead is also parametrically designed. It can be automatically updated based on the motor dimensions and the required resolution, speed and torque. A ratio of up to 


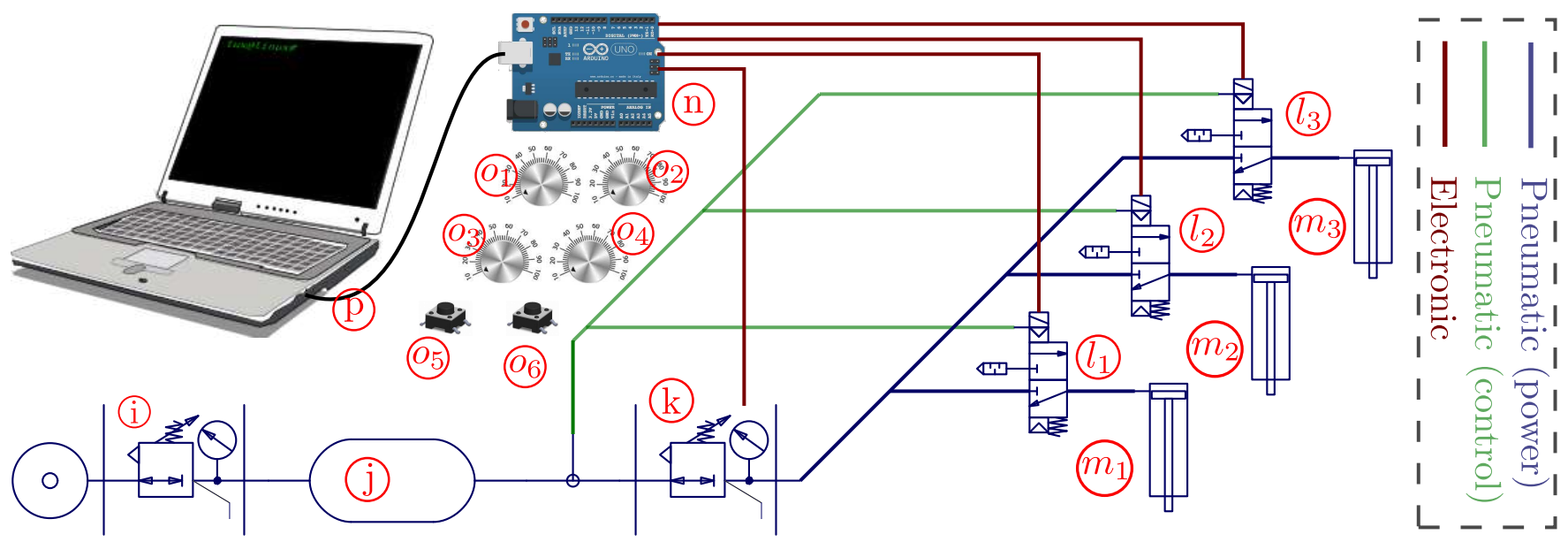

Fig. 3. A schematic illustration of the motor electro-pneumatic control system. Blue lines and green lines are power and control pneumatic tubes respectively. Red lines depict the digital electronic wiring. A manual proportional pressure regulator (i) controls the input pressure to the entire system and a reservoir $(j)$ reduces the fluctuations. A digital proportional pressure regulator $k$ controls the input pressure to the valves. $3 / 2$ pneumatic solenoid valves $\left(l_{1}\right), l_{2}$ and $l_{3}$ are externally piloted to reduce the effects of pressure change on their performance. Pistons $(m$, are representations of the pistons inside the motor. Solenoid valves and the digital proportional regulator are controlled via a micro-controller development board, Arduino $(n)$. The motor can be controlled in two different modes, speed control and position control via the button $O_{5}$. Potentiometers $O_{1}$, $O_{2}$, $O_{3}$ and $O_{4}$ control parameters: stepping frequency, signal width, position and input pressure, respectively. Button $O_{6}$ controls the direction of the rotation in speed control mode. A USB serial communication (P) is used for data logging, reprogramming the Arduino, and also controlling the mentioned parameters.

40 has been implemented for the gear-head, increasing the resolution from $120^{\circ}$ to $3^{\circ}$ in full-pitch drive and from $60^{\circ}$ to $1.5^{\circ}$ in half-pitch drive. This is smaller than the $3.3^{\circ}$ resolution reported by Stoianovici et al [6].

Additive manufacturing has been proven to be a promising production method for complex pneumatic motors [11]. A fuse deposition modeling (FDM) 3D printer (STRASYS Fortus 250MC) was used to build all the elements. No sealing, bearing or lubrication is included into the design. As a result the motor is highly affordable and can be used as a disposable unit. This expands the motor application to environments, such as surgery or food industry, where being hygienic is important. Airtightness in the motor was achieved through experiments to have the highest input pressure working range $(0.15 \mathrm{~mm})$ Fig. 7 . The entire structure of the actuator and gear-head are made of ABSplus-P430. Electromagnetic translucency of the motor is demonstrated using MRI tests. Spin Echo and Gradient Echo sequences were applied on a gelatin phantom in three different cases, without the motor, with the motor when it is off and when the motor is on. No measurable artifacts was observed in the images and signal to noise ratio (SNR) is unaffected Fig. 4. Please refer to the video accommodating this publication illustrating more details of design and experiments.

\section{ANALYSIS AND RESULTS}

A mathematical model of the motor is presented to describe the working principle of the motor. The power transmission system comprises multiple domains, including pneumatics, rigid body dynamics, and friction. Common approach in literature is to consider the pneumatics of the system as a reversible and adiabatic (isentropic) thermodynamic process [7]. Additionally, the effects of friction and stick-slip effect has not been included in the models. In this paper an experimental approach is presented.

\section{A. System Pneumatics}

Schematics of the experimental setup is illustrated in Fig. 5. Pressure-signal travel time in the tubes, as well as the force generated in a 3D-printed piston-cylinder, were measured. Tubes with an internal diameter of $4 \mathrm{~mm}$ and different lengths were tested under pressure signals with various magnitude, frequency, and duty-cycles. Pressure sensors (FESTO SPTE-P10R-S6-B-2.5K 571480) at both sides of the tubes measured the magnitude attenuation and phase shift of the pressure wave.

Experiments for different tubes with an internal diameter of $4 \mathrm{~mm}$, and lengths from $1 \mathrm{~m}$ to $8 \mathrm{~m}$, under pressures from 2 Bar to 7 Bar were performed. Using curve fitting, an empirical equation was developed to describe the response of tube to a step signal:

$$
P \approx P_{1}\left(1-e^{-0.05 e^{-l_{t} / 3}\left(t-\frac{10}{3} e^{l_{t} / 3}\right)}\right),
$$

where $P$ and $P_{1}$ are the pressure measured by the second sensor (at the end of the tube) and input pressure, and $t$ is time in milliseconds. Results of the experiment are shown in Fig. 6.

To measure the effects of clearance on force generated in a cylinder-piston, different pistons with various clearances from $0.05 \mathrm{~mm}$ up to $0.4 \mathrm{~mm}$ where tested. Experiments revealed a stochastic behavior caused by stick-slip effect. Moreover, dimensional inaccuracy of the 3D printing method adds to the volatility of the measurements. Due to the static friction a residual force after discharge was observed in the force sensor. Both steady-state response and residual force drastically diminish by an increase in clearance. Fig. 7 

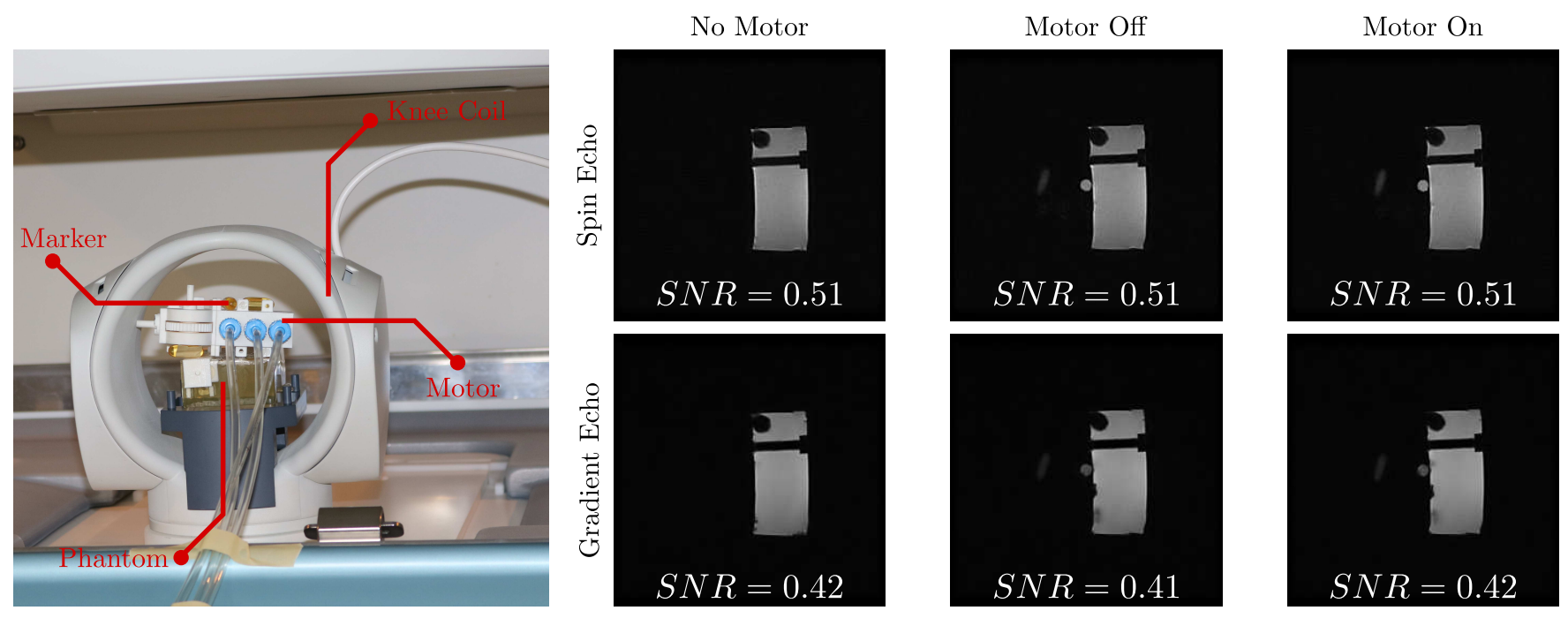

Fig. 4. Imaging experiments in MRI scanner 0.25T G-scan Brio, Esaote SpA (Genoa, Italy). Imaging parameters: TR $500 \mathrm{~ms}$, TE $30 \mathrm{~ms}$, Flip angle (FA) $90^{\circ}$, Number of acquisition (NSA) 1, FOV $20 \times 20 \mathrm{~cm}$, Reconstructed Resolution $0.78 \times 0.78 \times 5 \mathrm{~mm}$. Experimental data at GitHub repository [10].



Fig. 5. Schematics of the experimental setup for measuring the response of a valve, tube and cylinder piston system to a square wave pressure signal (Force sensor ATI Mini45). $P_{1}$ is the pressure of the reservoir, $P_{0}$ is the ambient pressure, $l_{t}$ and $D_{t}$ are the length and diameter of the tubes respectively, $D_{p}$ is the diameter of the piston.

shows an interpolation of the experimental data for the output force for different pressures and various clearances. As the graph illustrates when the clearance is smaller than about $0.2 \mathrm{~mm}$, stick-slip effect becomes effective and from there it drastically increases by decreasing the gap size. An increase in gap size also exponentially decreases the hydro-static force. The graph shows that the mechanical performance and working range of a cylinder piston are directly related. Smaller gaps lead to lower leakage and higher forces but static friction limits the working pressure range.

\section{B. Rigid-body model}

To have an understanding of the working principle of the motor and the interaction between friction and fluidic forces, a rigid body model of the system is developed. A freebody diagram of the motor is shown in Fig. 8, including the interacting forces and torques between the crankshaft, pistons and the cylinder. In an ideal mechanism, the torque

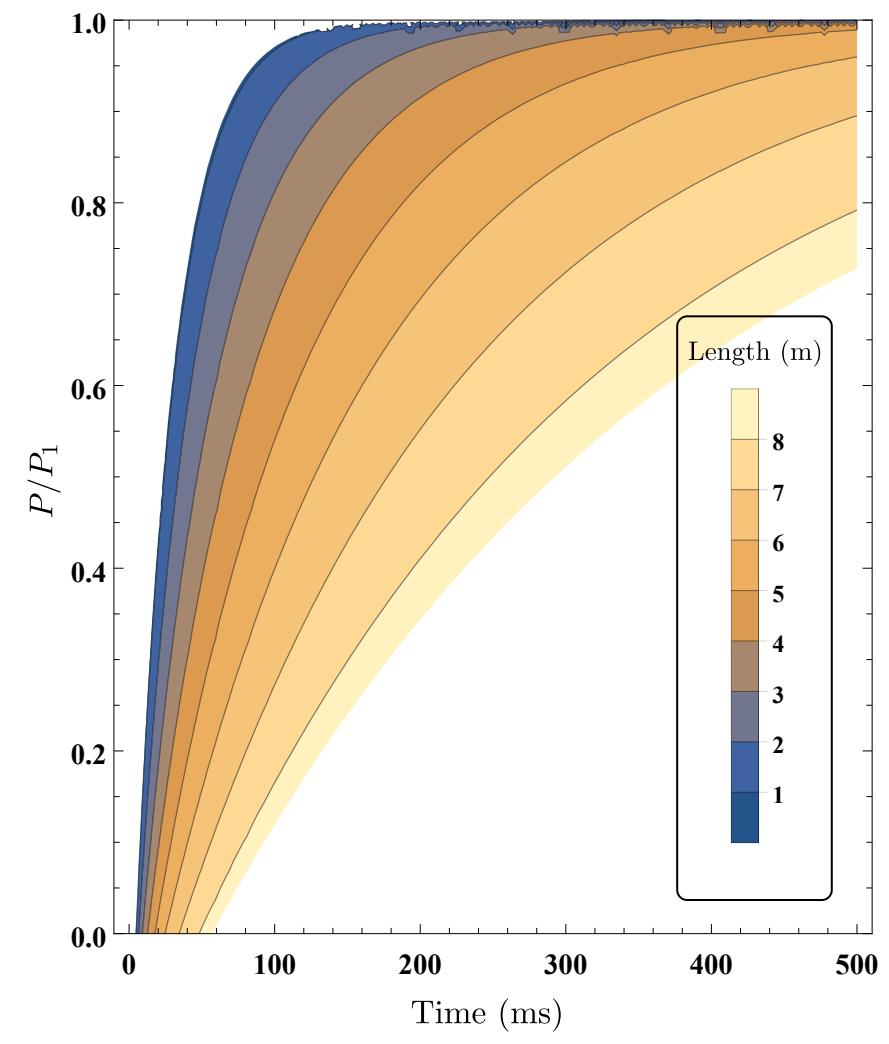

Fig. 6. Empirical model of the response of tubes with a $4 \mathrm{~mm}$ internal diameter and length from $1 \mathrm{~m}$ to $8 \mathrm{~m}$ to square wave pressure signals from 2 Bar to 7 Bar. and $P_{1}$ is the input pressure. Data-points at [10].

exerted by each piston can be calculated by the equation:

$$
\tau_{i}=P_{i} A_{p} s_{p} \sin \left(\theta_{i}\right)
$$

where $A_{p}$ is the piston surface area and $s_{p}$ is the stroke of the piston, $V_{c}=A_{p} s_{p}$ is the maximum volume of each cylinder. Assuming the distribution system is ideal and applies pressure only when the piston is moving down- 


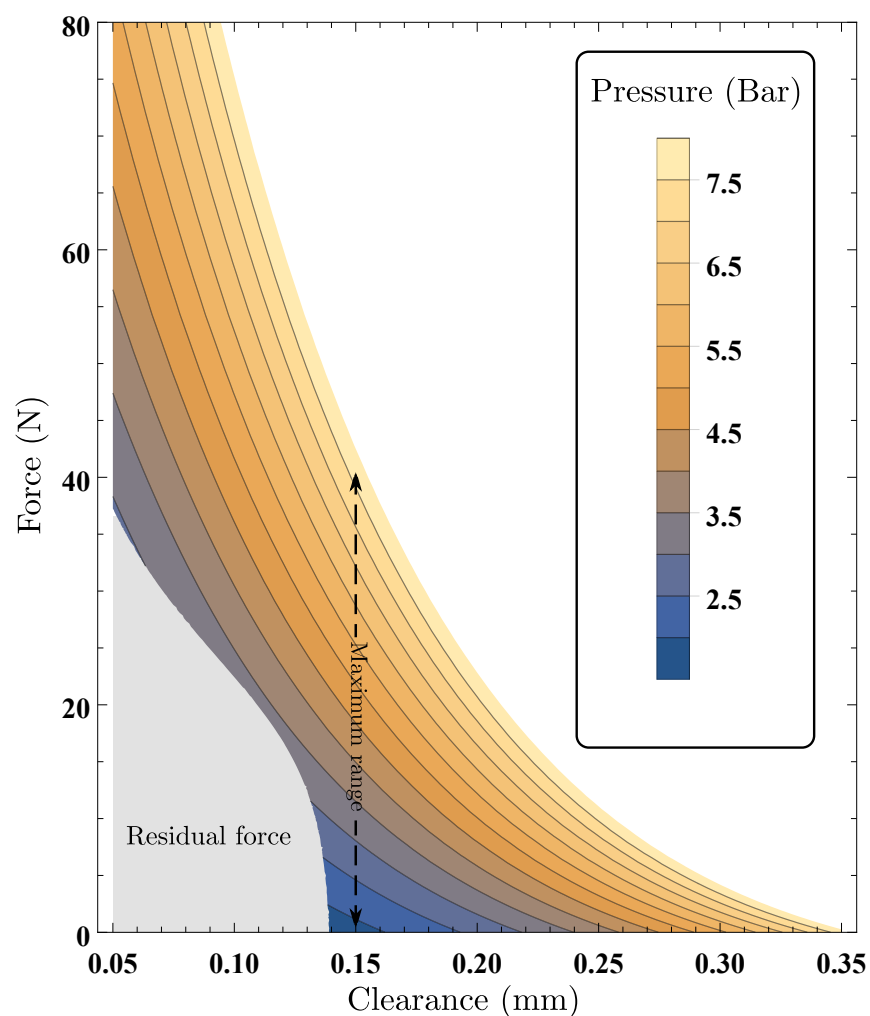

Fig. 7. An interpolation of measured steady-state force generated in the 3D-printed cylinder-piston illustrated in Fig. 5 with a cross section of $20 \times$ $20 \mathrm{~mm}^{2}$. Experimental data-points available in online repository [10].

wards, $P_{i}$ is a square wave signal. It can be calculated as $P_{i}=0.5 P_{i n}\left(1+\operatorname{sgn}\left(\sin \left(\theta_{i}\right)\right)\right)$, where $\operatorname{sgn}(x)$ is the sign function. The average torque during one rotation of the motor can be calculated as:

$$
\tilde{\tau}=\frac{1}{2 \pi} \int_{0}^{2 \pi} \sum_{i=1}^{1} P_{i} A_{p} s_{p} \sin \left(\theta_{i}\right) \mathrm{d} \theta_{1}=\frac{3 V_{c} P_{i n}}{\pi},
$$

This is the maximum torque if there was an ideal motor that could convert the total pneumatic energy into mechanical power. However, in reality, leakage and friction dissipate a large portion of the power, as was observed during the experiments. Including the effects of friction, considering planar model of the motor, there are three conservation of momentum equations for each element. Angular and linear equations of momentum conservation of the crankshaft are:

$$
\begin{gathered}
J_{s} \ddot{\theta}_{s}=\sum_{i=1}^{3} \tau_{i}-\tau_{\text {ext }}-\tau_{f s c}, \\
\vec{F}_{s c}=\sum_{i=1}^{3}\left(\begin{array}{c}
F_{n s p_{i}} \\
F_{f s p_{i}}
\end{array}\right),
\end{gathered}
$$

where $J_{c}$ is the angular inertia of the crankshaft, and $\tau_{i}$ is the total torque applied from each piston on the crankshaft, as calculated below:

$$
\tau_{i}=F_{n s p_{i}} y_{i}-F_{f s p_{i}} x_{i}-\tau_{f s p_{i}}
$$
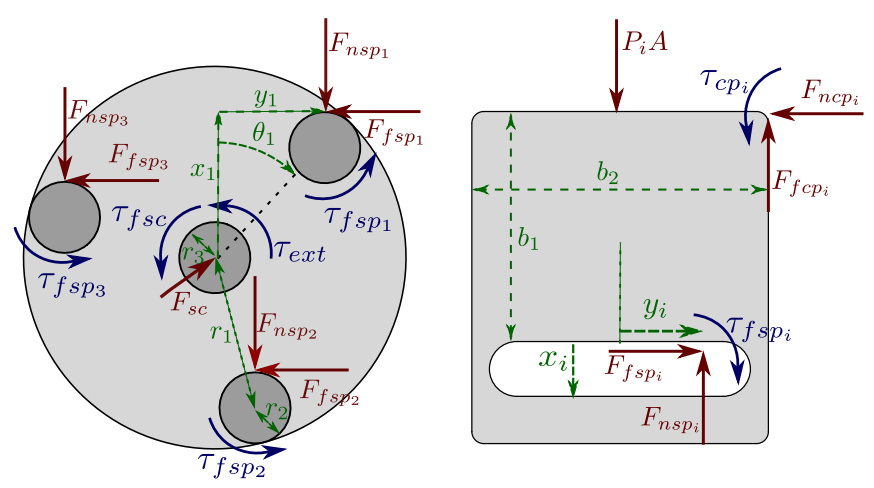

Fig. 8. Free-body diagram of a piston and the crankshaft. $\theta_{i}$ is the angle between crank-pin $i$ and vertical axis in clockwise direction calculated as $\theta_{i}=\theta_{1}+(i-1) \frac{2 \pi}{3} . \tau_{f s c}$ is the frictional torque between the crankshaft and cylinder, $y_{i}=r_{1} \sin \left(\theta_{i}\right)$ and $x_{i}=r_{1} \cos \left(\theta_{i}\right)+r_{2}$ are coordinates of the assumed contact points between crank-pins and each piston. $F_{n s p_{i}}$ is the vertical normal force and $F_{f s p_{i}}$ and $\tau_{f s p_{i}}$ are horizontal force and torque due to friction, between the pistons and the crankshaft, $\vec{F}_{s c}$ is the normal force from the cylinder on the crankshaft, $F_{n c p_{i}}$ and $F_{f c p_{i}}$ are the normal and friction forces from cylinder on the pistons.

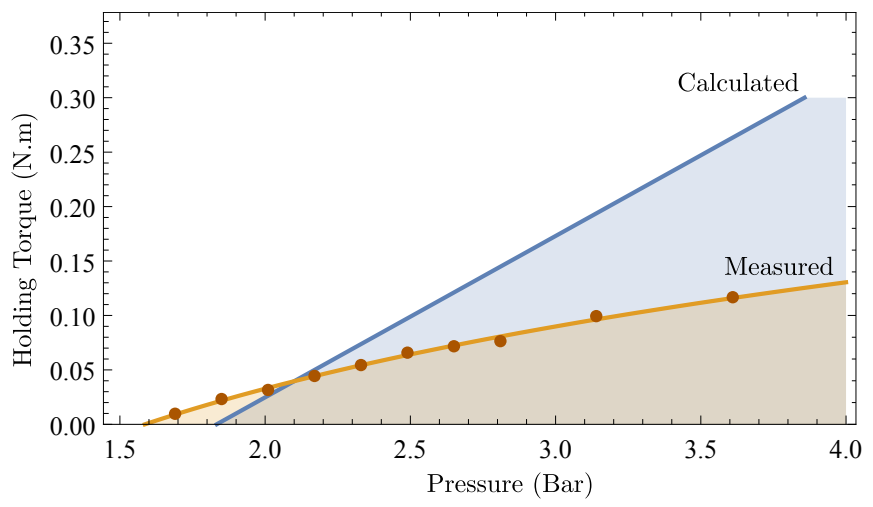

Fig. 9. Calculated and measured minimum holding torque for the motor (C) illustrated in Fig. 2, including the logarithmic interpolated trend-line. Experimental data-points are available in an online repository [10].

The conservation of momentums equations for the pistons are:

$$
\begin{gathered}
m_{p} \ddot{x}_{i}=P_{i} A_{p}-F_{f c p_{i}}-F_{n s p_{i}}, \\
F_{f s p_{i}}=F_{n c p_{i}}, \\
\tau_{f s p_{i}}+F_{n c p_{i}}\left(\frac{b_{2}}{2}-y_{i}\right)=F_{f s p_{i}} b_{1}-P_{i} A_{p} \frac{b 2}{2}+\tau_{c p_{i}},
\end{gathered}
$$

where $m_{p}$ is the linear inertia of a piston. Fig. 9 shows the calculated and measured minimum holding torque for the motor (c) from Fig. 2. The difference between measured and calculated holding torques is mainly due to the simplified Coulomb frictional model.

\section{Stall Curve}

Stall curve indicates the working range of a PSM. The stall curve changes with input pressure, length and diameter of the tubes, as well as the duty-cycle, and frequency of the input pressure waves. For any given Input pressure and frequency, there is a maximum external torque the motor can sustain 


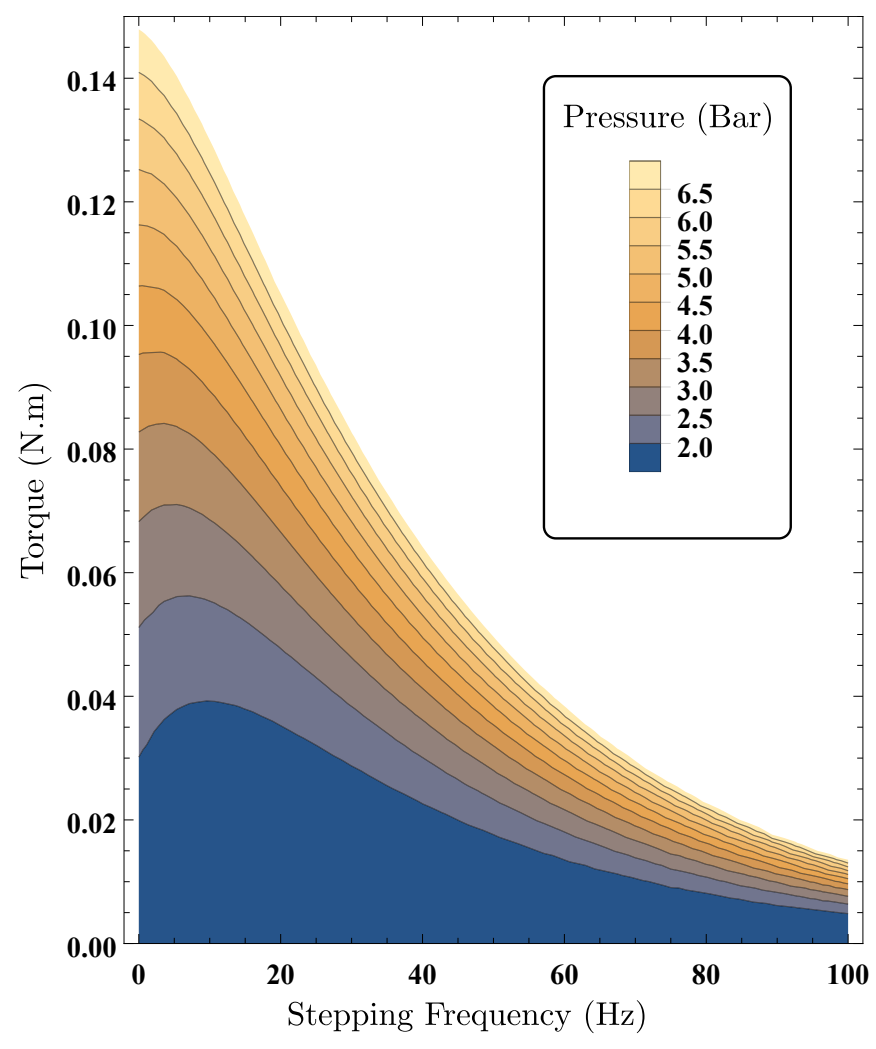

Fig. 10. Stall curve of the motor (c) gap size: $0.15 \mathrm{~mm}$, piston surfacearea: $15 \times 15 \mathrm{~mm}^{2}$, crankshaft radius: $r_{2}=3 \mathrm{~mm}$ (Fig. 8), Gear-head ratio: 1:40, Input pressure from 1 to $8 \mathrm{Bar}$, stepping frequency from $0 \mathrm{~ms}$ to $100 \mathrm{~ms}$, pulse-width: $9 \mathrm{~ms}$, Tube length: $3 \mathrm{~m}$ and internal diameter: $4 \mathrm{~mm}$. Experimental data are available in an online repository [10].

without skipping any steps. Exceeding this safe torque leads to stochastic behavior due to the stick-slip effect, called ratcheting torque. Similarly, there is a maximum exciting frequency at any given torque and input pressure, called the throttling frequency. Using dynamometers has been the common approach in literature for torque measurement [6]. However, due to the abrupt behavior of pneumatic actuation, accurate measurement is challenging. In this experiment, a pulley mechanism was built, lifting weights to measure stall torque of the motors. The maximum weight the motor could lift without skipping any steps was recorded. Different experiments were implemented that measured the effects of pressure input, stepping frequency, speed, pulse-width, external torque, length and diameter of the tubes, as well as the effect of lubrication on motor performance. Fig. 10 shows the holding torque of the motor (c) combined with gearhead (d) at different input pressures and stepping frequencies. The surface area of the pistons is $15 \times 15 \mathrm{~mm}^{2}$ with a gap size of $0.15 \mathrm{~mm}$ to achieve the highest pressure range. A constant pulse width of $9 \mathrm{~ms}$ was used, minimum required to activate the valves. Observable nonlinearity can be ascribed to leakage and friction. At higher pressures leakage increases, lowering the mechanical performance of the motor.

As can be seen the minimum activation pressure slightly improves at higher frequencies showing the effects of static friction. However, at higher speeds viscose friction, throttling of the pressure waves in the tubes and valves malfunction cause the torque to drop rapidly.

\section{CONCLUSIONS AND FUTURE WORK}

In this paper a new paradigm of parametrically designed actuators is introduced. A miniaturized version of the rotational pneumatic stepper motor with a total volume of $4.6 \mathrm{~cm}^{3}$ is also presented. The motors are able to sustain a no-load-speed of $800 \mathrm{rpm}$. Using a worm-drive a resolution of $3^{\circ}$ at full-pitch was achieved. The entire motor and the gear-head are 3D-printed with no bearing, sealing or lubrication. This demonstrates the possibility of making volumetric pneumatic actuators solely with additive manufacturing. The motors are highly affordable and can be used as a disposable part in medical applications. The motors are completely MRI translucent and no artifacts or distortions were observed in the tests. The motor is back-drivable, as a result it provides a safe actuation method for surgical applications. A robotic mechanism actuated by PenuAct motors is safe because it can be used as a passive tool-holder in emergency situations.

Measuring the effects of clearance in a 3D-printed cylinder and piston, on pneumatic leakage and generated force is one of the practical contributions of this work.

Furtherer studies are are required to overcome some shortcomings including acoustic noise, mechanical vibration, mechanical performance, stick-slip effect and durability.

\section{REFERENCES}

[1] R. Gassert, A. Yamamoto, D. Chapuis, L. Dovat, H. Bleuler, and E. Burdet, "Actuation methods for applications in MR environments," Concepts in Magnetic Resonance Part B: Magnetic Resonance Engineering, vol. 29B, no. 4, pp. 191-209, 2006.

[2] K. Chinzei, R. Kikinis, and F. A. Jolesz, MR Compatibility of Mechatronic Devices: Design Criteria. Berlin, Heidelberg: Springer, 1999, pp. 1020-1030.

[3] K. Suzumori, K. Hori, and T. Miyagawa, "A direct-drive pneumatic stepping motor for robots: designs for pipe-inspection microrobots and for human-care robots," in Proc. IEEE Int. Conf. on Robotics and Automation (ICRA), vol. 4, May 1998, pp. 3047-3052.

[4] K. Suzumori, T. Hashimoto, K. Uzuka, and I. Enomoto, "Pneumatic direct-drive stepping motor for robots," in IEEE/RSJ International Conference on Intelligent Robots and Systems, vol. 2, October 2002, pp. 2031-2036.

[5] E. Taillant, J.-C. Avila-Vilchis, C. Allegrini, I. Bricault, and P. Cinquin, CT and MR Compatible Light Puncture Robot: Architectural Design and First Experiments. Berlin, Heidelberg: Springer, 2004, pp. 145152 .

[6] D. Stoianovici, A. Patriciu, D. Petrisor, D. Mazilu, and L. Kavoussi, "A new type of motor: Pneumatic step motor," IEEE/ASME Transaction on Mechatronics, vol. 12, no. 1, pp. 98-106, Feb 2007.

[7] H. Sajima, I. Sato, H. Yamashita, T. Dohi, and K. Masamune, "Two-DOF non-metal manipulator with pneumatic stepping actuators for needle puncturing inside open-type MRI," in World Automation Congress, Sept 2010, pp. 1-6.

[8] Y. Chen, K.-W. Kwok, and Z. T. H. Tse, "An MR-conditional hightorque pneumatic stepper motor for MRI-guided and robot-assisted intervention," Annals of Biomedical Engineering, vol. 42, no. 9, pp. 1823-1833, 2014.

[9] Y. Chen, C. D. Mershon, and Z. T. H. Tse, "A 10-mm MR-conditional unidirectional pneumatic stepper motor," IEEE/ASME Transaction on Mechatronics, vol. 20, no. 2, pp. 782-788, April 2015.

[10] "GitHub repository for experimental data-points." [Online]. Available: https://goo.gl/9o2p6u

[11] Y. Wei, Y. Chen, Y. Yang, and Y. Li, "Novel design and 3D printing of nonassembly controllable pneumatic robots," IEEE/ASME Transactions on Mechatronics, vol. 21, no. 2, pp. 649-659, April 2016. 